\title{
A new approach for mountain areas cartography
}

\author{
Loïc Gondol, Arnaud Le Bris, François Lecordix \\ Institut Géographique National (IGN France) \\ 2-4 Avenue Pasteur \\ 94165 SAINT-MANDE Cedex - France \\ loic.gondol@ign.fr, arnaud.le-bris@ign.fr, francois.lecordix@ign.fr
}

\begin{abstract}
From now on, the French National Mapping Agengy (IGN France) is set up with the BD TOPO ${ }^{\circledR}$. This is a topographic vector database that covers the whole national territory. IGN has decided to product base maps at $1: 25 \mathrm{k}$ and $1: 50 \mathrm{k}$ from this database. On topographic mountain maps, rocks areas are among the most difficult map elements to represent, dealing with digital cartography. In the past, they were drawn manually by experienced cartographers, using graphic means and working with aerial photographs. Nowadays, we need to focus on two points with a digital approach. The first one is the detection and an automated classification of concerned areas. The next one is the development of an adapted cartographic representation of rocks and screes areas. This article presents the first results on these problems. As far as possible, we aim at having automated high mountain cartography with lower production costs. Also, we would like it to be as expressive as it was in previous maps. This is to keep the same cartographic quality of the current base map at $1: 25 \mathrm{k}$ and $1: 50 \mathrm{k}$.
\end{abstract}

Keywords: cartography, representation, mountain, classification, data fusion 


\section{Context}

\subsection{The BD TOPO®}

The French National Mapping Agency has decided to create a topographic database for several years. This is the BD TOPO ${ }$. This vector database provides some geographic information about road, rails, electric and hydrographical networks, but also about buildings, administrative boundaries, toponymy, land use and relief. The first version of the BD TOPO ${ }^{\circledR}$ (V1) was mainly acquired by digital restitution, and it included all the needed information for the map at 1:25k. However it required a huge workload and therefore too much time to realise this database on the whole territory. In 2000, IGN France chose a new lighter specification for the BD TOPO ${ }^{\circledR}$ that allowed its completion on the whole territory by the beginning of 2007.

Beyond various applications linked to GIS, the BD TOPO ${ }^{\circledR}$ is also useful to derive the base map, produced and diffused by IGN France. The base map includes a topographic map at $1: 25 \mathrm{k}$ and another one at $1: 50 \mathrm{k}$. Since 1993,450 out of 1800 maps at 1:25k have entirely been produced from the BD TOPO® $\mathrm{V} 1$ on several areas of the national territory.

Nevertheless, up to now, none map covering a high mountain area has been done from the BD TOPO ${ }^{\circledR}$ V1. In fact, the cartographic representation of these areas is knotty dealing with a digital production. On high mountain areas, all current base map versions come from revisions of former versions drawn manually years ago. They are not originally from BD TOPO ${ }^{\circledR}$. As a result, we currently have 2 distinct processes to collect data, update the database and the topographic map.

\subsection{The New Base Map Project}

In 2004, IGN France decided to launch the New Base Map Project in order to derive the base map from the BD TOPO ${ }^{\circledR}$ with its new specifications, and to reduce update costs of next base map versions. The process is planned to work on the whole territory, and then on high mountain areas.

Among other issues, this project has to provide solutions to retrieve the needed information for the map that is lacking in the new specifications of the BD TOPO®. In particular, the land use is one of the main incomplete themes. This recovery issue is especially perceptible in high mountain areas where a paramount part of the map information deals with mountain 
land use: rocks, screes, glaciers... Without these themes, mountain maps would appear uncluttered and could not satisfy users.

It is not enough to recover the needed information in high mountain areas. Indeed, another issue arises that has to be carried out by the New Base Map Project. It deals with the digital representation of these specific themes, in order to get the most expressive possible result on concerned areas.

\subsection{From manual to digital}

During the history of cartography, a lot of solutions have been tested for the cartographic representation of mountain areas on topographic maps. In (Imhof 1958), a literature review of these solutions is presented. The relief aspect often comes from a combination of different techniques: hill shading, graphic means, contour lines with a colorimetry depending on areas (glaciers, rocks...), etc. But up to now, the solutions that have been carried out and the best provided results are only the outcome of traditional graphic techniques. At present, to edit topographic maps (at 1:25 000 or 1:50 000) in high mountain areas, the National Mapping Agencies are not able to produce numerically new maps without some elements which were drawn manually in the past by traditional cartographers. Figure 1.a. shows an extract of an IGN topographic map at 1:25k, where an example of this cartographic result on the Alps is shown.

In the beginning of the 2000's, IGN France launched the first studies in the purpose of considering the feasibility of substituting digital solutions for traditional methods (Le Men et al. 2002). These works focused on the automatic extraction of map needed information (rocks areas, screes, glaciers) and provided the first steps for data representation. These study results provided in a digitally manner are presented on figure 1.b..

The New Base Map Project has been continuing these first works. In particular, it looks at improving final cartographic results. Dealing with data retrieval, the MATIS, which is a research laboratory of IGN in image processing, has been carrying out the study. 

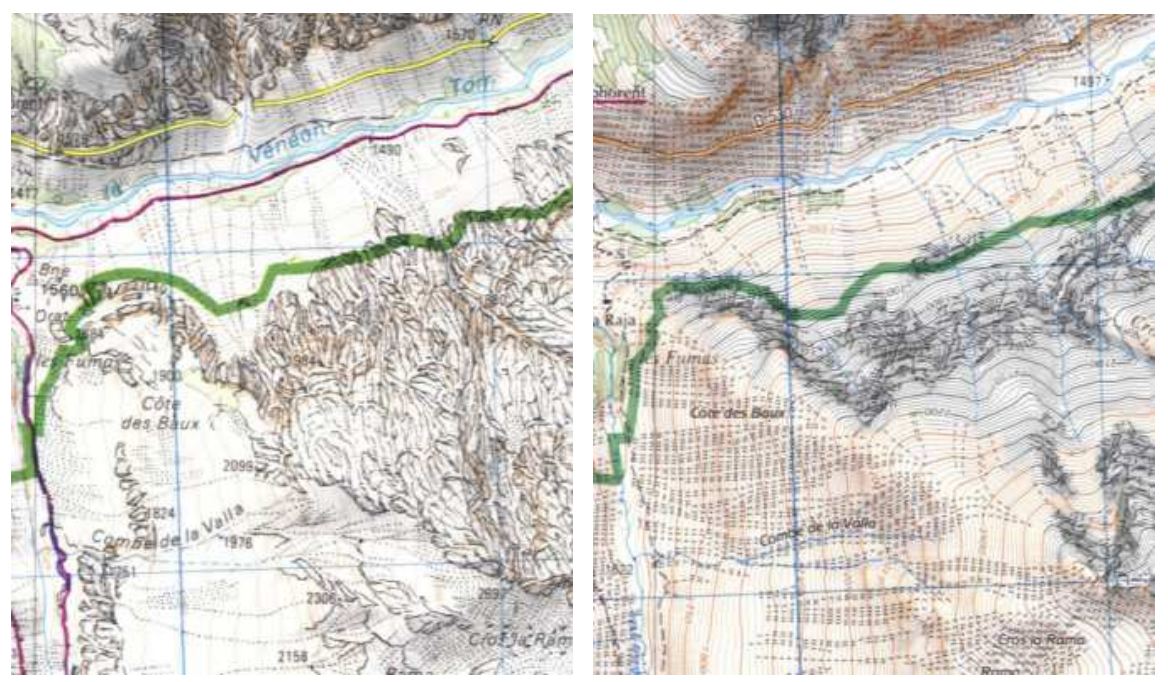

Fig. 1. On the left (fig.1.a.) IGN present handmade topographic map at 1:25k and on the right (fig.1.b.) first map digitally obtained by (Le Men et al. 2002) on the same area.

\section{Information extraction}

\subsection{Problems and proposed solutions}

Two ways of getting back landcover information that lacks from BD TOPO ${ }^{\circledR}$ but is necessary to obtain the topographic map are possible: it can be extracted either from aerial (ortho-)images (through (semi-)automatic classification) or from present maps. As the cartography of these missing landcover themes is not up to date in present maps, the first solution has been chosen (Le Men et al. 2002). Furthermore, it could also be used afterwards for map updating. So, the chosen solution consists in extracting landcover information out of aerial orthophotos from IGN France's orthoimage database BD ORTHO ${ }^{\circledR}$ through a supervised classification method. Nevertheless, it must be said that extracting such information from satellite images with bands dedicated to remote sensing (such as in (Paul 2003)) could have been possible too, but in the present case, using aerial data is interesting since it is available and captured regularly by IGN.

The only lacking landcover themes necessary to make the map are rocks, screes and glaciers. However the classification legend must contain 
more items than these three seeked lacking themes to obtain a landcover classification of whole mountainous areas. That's why it consists of the six following classes: rocks, screes, glaciers, forests, high mountain pastures and water areas.

In high mountain areas, landcover extraction using aerial photographs is bothered by several phenomena:

- Shady areas are often very large.

- The radiometry of a same theme can greatly vary within the image. This phenomenon can be due to illumination variations related to the rough relief, but it can also be "artificial" (since the image is in fact a mosaic of orthorectified aerial images which have not been captured at the same time and have undergone several different radiometric treatments) or natural (as in case of changes in geology inside the area).

- Some of the landcover themes have a very close radiometry: they look like each other on the image as for instance some screes (especially riverbed screes) which are almost as light as glaciers or lakes which are often difficult to distinguish from rocks in shadow, or even rocks and screes. This phenomenon is increased by the variations of radiometry explained above.

As a consequence, image information is not sufficient to obtain a correct classification. Nevertheless, the introduction of complementary information in the classification process can improve the result. Two kinds of external knowledge are useful:

- Mountainous landcover is strongly related to the relief, it means to altitude, slope and orientation. Those variables can be easily computed from a Digital Terrain Model and used to obtain a probability to find the different themes.

- Knowledge from another database can also be used. In the present case, European database CORINE Land Cover 2000 (CLC2000) dealing with land use has been used. It is more generalized than the base map since the smallest mapped area is 25 hectares and the better scale to use the data base is 1:100k (Bossard et al. 2000) (fig.2.). Its legend is different too, with a varying semantic precision : for example, different kinds of forests are separated, whereas rocks and screes are contained within a unique class. Besides, in other cases, some CLC2000 classes describe intermediate situations between several of our classes: "forest and evolving shrubby vegetation" is linked both to forest and pastures. 


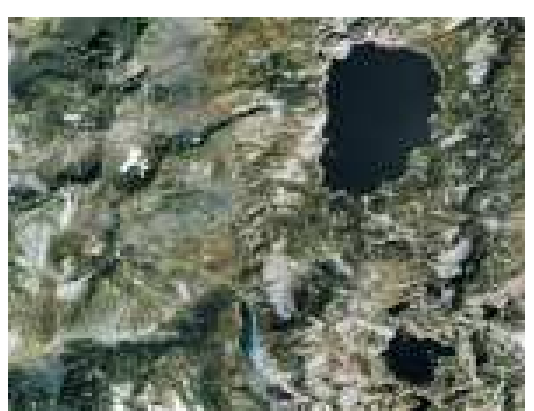

Fig. 2. CLC2000 is a more generalized database.

As shadow areas are important on the image, they must be taken into account to obtain a classification of the whole area. A first way to achieve this could consist in correcting the radiometry in shadow areas (after having detected them). However, it would be limited by uncertainties concerning the DTM and the fact an orthoimage (it means a mosaic of merged orthorectified aerial photographs captured at different times and having undergone different radiometric treatments) is used. As the main problem with shadows is the fact that the radiometric model of a class will be completely different in shadow and in light, a second solution consists in dividing each class " $\mathrm{C}$ " in two classes " $\mathrm{C}$ in shadow" and "C in light" so that two distinct models are computed for each theme. Of course, these two classes are aggregated at the end of the classification. The second solution has been retained for the present method. Nevertheless, a method using radiometric correction in shady areas has been successfully developed by (Le Men et al. 2002) during the first studies about this issue.

Even though the available information is not sufficient to precisely detect and correct shadows, it can be used to compute an approximate prior probability for each pixel of the image to lie in shadow knowing the DTM (since the beginning and final time of data capture of all images are known even if the exact capture time of each pixel of the orthoimage is not precisely known). It could help to discriminate dark themes (such as water) in light from themes in shadow.

New channels can be computed from the original bands (red-green-blueinfrared) of the orthoimage. Associations of these derived channels can lead to better results since some channels are more efficient than others to separate some classes.

\subsection{Method}

The landcover extraction method consists of two steps. 
- The orthoimage is firstly segmented into homogeneous regions (fig.3). A description of the used segmentation tool can be found in (Guigues 2004) and (Guigues et al 2006).

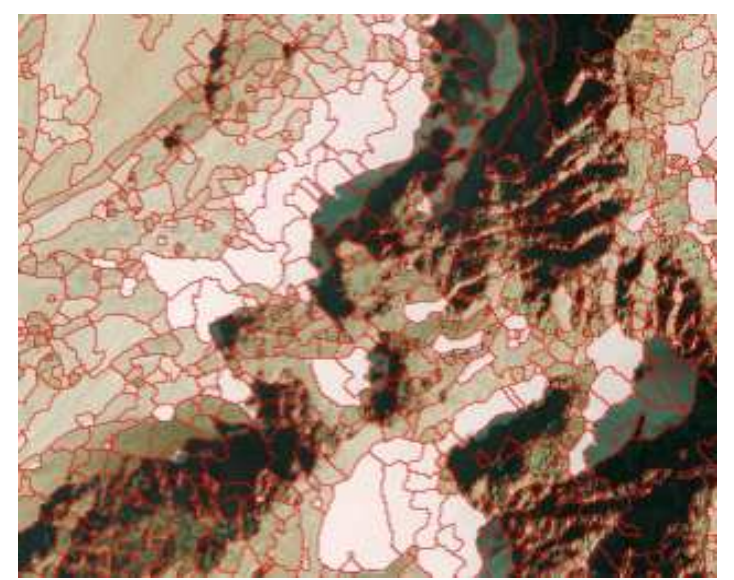

Fig. 3. The image is segmented in homogeneous regions.

- Segmentation's regions are then classified by the tool presented in (Trias-Sanz 2006) and (Trias-Sanz and Boldo 2005). The way image information is used in the classification process consists of two steps :

- A model is computed from training data captured by an operator. First, for each class, the best parameters of several statistical distributions (such as gaussian, laplacian laws but also histograms (raw or obtained by kernel density estimation)...) are computed to fit to the radiometric $\mathrm{n}$-dimensional histogram of the class (with $\mathrm{n}$ standing for the number of channels used in the classification). Then the best model is selected thanks to a Bayes-Information-Criterion (Schwarz 1978) allowing to choose an alternative between fit to data and model complexity (The more complex the model is, the more degrees of freedom it has, the better it is able to describe training data, but the most it is also at risk to "stick" too much to training data without describing the whole groundtruth as well as a simpler one.) Once this model has been computed, it becomes possible to compute the probability that a pixel $\mathrm{s}$ has a certain value $\mathrm{I}(\mathrm{s})$ if it belongs to class $\mathrm{c}$, it means to obtain $\mathrm{P}_{\text {radiomerricmodel }}(\mathrm{I}(\mathrm{s}) \mid \mathrm{c}(\mathrm{s})=\mathrm{c})$ with $\mathrm{c}(\mathrm{z})$ standing for region or pixel "z's class".

- In the present case, a MAP per region classification algorithm is used since it allows to take easily into account external information (from relief, from CLC2000 and concerning shadow probability in the pre- 
sent case) as prior probabilities. Classifying regions prevents from obtaining too noisy results. With this classification method, the label $c_{0}(R)$ given to a region $\mathrm{R}$ is its most probable class according to the radiometric model previously estimated and prior probabilities. Hence, $c_{0}(R)$ is the class $\mathrm{c}$ that maximizes the following func-

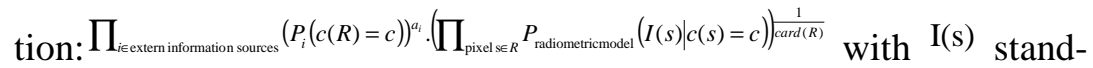
ing for the radiometry vector of pixel $\mathrm{s},{ }^{\mathrm{c}(\mathrm{z})}$ meaning region or pixel "z's class" and $\mathrm{P}(\mathrm{c}(\mathrm{z})=\mathrm{c})$ standing for the probability for pixel or region $\mathrm{z}$ to belong to class $\mathrm{c}$. The ${ }^{\mathrm{a}_{\mathrm{i}}}$ terms stands for weight parameters balancing the different prior probability sources.

External information is then introduced as prior probabilities in the classification process (Le Bris and Boldo 2007). This requires an interpretation of this knowledge in terms of probability.

As previously said, in mountainous areas, landcover is strongly related to relief. Thus it depends on altitude, slope and orientation making it possible to compute the probability to find the different themes knowing these three parameters. Such a model is proposed by (Le Men et al. 2002) from physical geography knowledge (such as such as the lowest and highest limits of landcover themes...) presented in (Elhai 1968) and (Lacambre 2001). It consists of two distinct models (made of piecewise linear mathematical functions) depending on altitude and slope (figure 4). Orientation has a significant influence only on forests and glaciers. Thus it is taken into account only for these themes as a modification of the altitude.
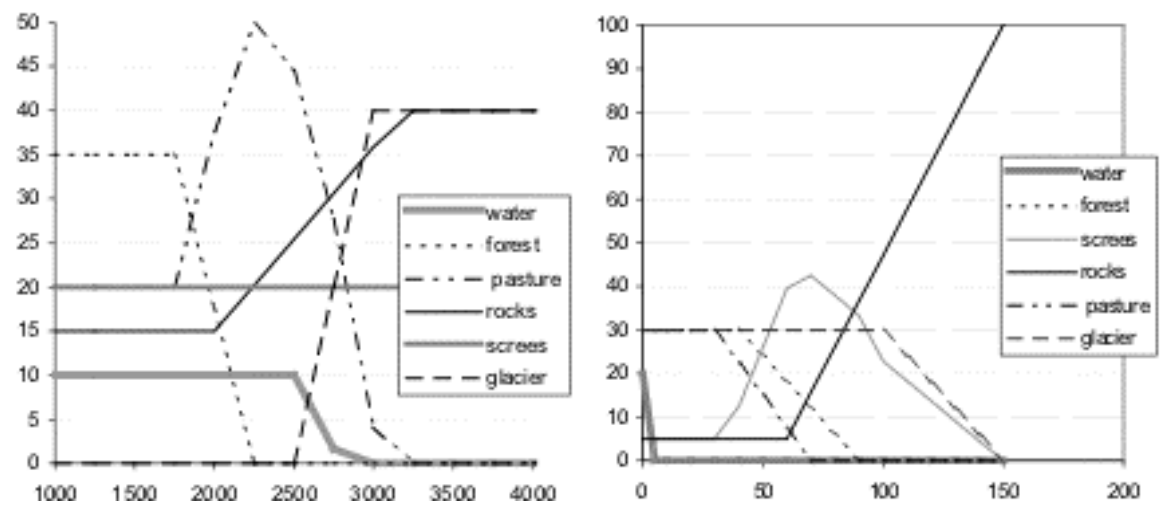

Fig. 4. On the left [right], probability of finding themes knowing altitude [slope].

CLC2000 information must also be introduced into the classification process. As this database is more generalized than expected results, a CLC2000 area can contain several classification themes. Besides, as its le- 
gend is different, several themes of our classification can be related to one CLC2000 item, and vice versa. Therefore the introduction of information from CLC2000 in the classification process must deal with those two kinds of uncertainties. Therefore CLC2000 is interpreted in terms of probability with an empirical probability model: for each CLC2000 item $T_{C L C 2000}$ and for each classification class $T_{\text {classif }}$, a probability value $P\left(T_{\text {classif }} \mid T_{C L C 2000}\right)$ is empirically defined. For instance, for CLC2000 class "forest and evolving shrubby vegetation", the probability to find water and glaciers areas is null but equals $77 \%$ for forests, $20 \%$ for pastures, $1 \%$ for rocks and $2 \%$ for screes.

Shadow knowledge is also taken into account as probabilities of being in shadow given the relief, i.e. the DTM, and the interval of image capture time.

A balance between these different sources is applied, allowing to give more or less strength to some of them but also to modify the generalisation level of the result (the higher CLC2000 weight is, the more generalised results are).

\subsection{Results}

This method has been tested in three study cases. The first test zone, located in the Alps, near St-Christophe-en-Oisans, has already been the test zone of the preliminary study (Le Men et al. 2002). All the classification themes are present, but only an old 3-bands orthoimage made from argentic scanned photographs is available there. Due to very important radiometric variations within the image, it was sometimes difficult, even for an operator, to identify themes. The second test area is located in the Pyrenees, around the Ossau-peak. Orthophotos have been produced from digital pictures in red-blue-green-infrared. All classification themes, except significant glaciers, were present. The third test zone has been chosen in the Alps near Modane, where 4-bands orthoimages (captured by a digital camera) are available and where all the classification themes are present. This area is interesting since available data (orthoimage and DTM) corresponds to what would be used in a production context. Furthermore, digital topographic data captured by operators in the 1990's are available there and can be used to evaluate the classification results.

Results have been visually evaluated (on whole images) revealing no major errors. Nevertheless, over detection can occur concerning glaciers 
on Modane area : snow and glaciers can be mistook. Furthermore, most of the classification regions have a meaningful size to be relevant on the map.

They have also been numerically (on smaller test zones in the image) evaluated by computing confusion matrices comparing test data captured by an operator to classification results. Nevertheless, it is sometimes difficult to evaluate the obtained results since even a human operator can find it hard to discriminate landcover themes in some parts of the test zones. These results can be seen in table 1 .

Table 1. Evaluation of the classification results on the three test zones. us-ac corresponds to the probability for a classified pixel to be really part of its class whereas pr-ac corresponds to the probability for a ground pixel belonging to a given class to be well classified.

\begin{tabular}{lcccccc}
\hline & $\begin{array}{c}\text { St-Christophe-en- } \\
\text { Oisans (Alps) }\end{array}$ & \multicolumn{2}{c}{$\begin{array}{c}\text { Ossau } \\
\text { (Pyrenees) }\end{array}$} & \multicolumn{2}{c}{$\begin{array}{c}\text { Modane } \\
\text { (Alps) }\end{array}$} \\
\hline & \multicolumn{5}{c}{ With complementary knowledge } \\
\hline Us-ac & pr-ac & us-ac & pr-ac & us-ac & pr-ac \\
\hline Water (Lakes) & $/$ & $/$ & 100.0 & 76.4 & 98.3 & 40.6 \\
Forest & 81.9 & 65.2 & 89.0 & 95.8 & 98.7 & 88.8 \\
Pasture & 71.1 & 52.2 & 96.3 & 85.1 & $/$ & $/$ \\
Rocks & 76.4 & 69.9 & 71.0 & 87.2 & 54.2 & 76.5 \\
Screes & 54.7 & 73.3 & 88.0 & 83.1 & 68.9 & 52.3 \\
Glaciers & 58.6 & 69.5 & 98.3 & 72.2 & 91.5 & 67.3 \\
\hline Well classified pixels & $67.0 \%$ & $87.4 \%$ & $75.6 \%$ \\
\hline & \multicolumn{7}{c}{ Only image information } \\
Well classified pixels & \multicolumn{7}{c}{$75 \%$} \\
\hline
\end{tabular}

Several parameters have been tested. In particular, these tests have shown that almost equivalent satisfying results are provided by several channels associations such as the intensity-hue-NDVI (NormalizedDifference-Vegetation-Index) one, or the three channels of the KarhunenLoève colorimetric space (Wang et al. 2003). The significance of the external knowledge and the importance of the balance between them have also been proven.

At the end of the classification, classes "c in shadow" and "c in light" are aggregated in a single class "c". Then, only themes lacking from BD TOPO ${ }^{\circledR}$ (rocks, screes and glaciers) are kept since information about other themes is already available. So all needed landcover information is then available to make the $1: 25 \mathrm{k}$-scale topographic map and now needs to be mapped. 


\section{Cartographic representation}

\subsection{Further processes for cartographic representation}

Classification results can not be directly used to draw the map: they have to be both simplified and enriched for cartographic requirements. Thus additionnal processes have been designed to post-process them.

On one hand, too small areas (without cartographic meaning) are filtered out because they would be irrelevant and unreadable at a given scale $(1: 25 \mathrm{k})$.

On the other hand, screes, rocks and glaciers areas form the heart of the cartographic representation. They delineate areas we are interested in the present study. More particularly, rocks and screes correspond to rough relief areas: their cartography should therefore also be a cartography of relief, an interpretation of terrain characteristics. Thus, classification results concerning these themes have to be enriched with additionnal information extracted from DTM to improve the final cartographic representation.

Firstly, rocks and screes areas are split up depending on the slope value in order to associate a proper symbolisation to these different kinds of areas. A threshold allows to distinguish steep slopes from gentle ones: a $100 \%$ slope is the limit for rocks areas whereas it is $50 \%$ slope for screes ones.

A rocky areas classification following slope orientation criterion is then processed. As previously, it aims at differentiating the cartographic representations depending on terrain characteristics. On one hand, this classification has to contain enough details to illustrate as best as possible the terrain diversity and complexity. On the other hand, areas have to be big enough to be readable on a map at $1: 25 \mathrm{k}$. After several tries, the creation of 18 classes at $20^{\circ}$ regular intervals has appeared to be the best choice in the present case. However, be careful not to generalise without any cares this result. The first point is to respect qualitative criteria explained above. Finally, rocks areas are divided in 36 different classes, depending both on slope orientation and slope value.

Screes areas characterised by a steep slope will be drawn with growing points along the slope. Therefore, main slope lines (i.e. the symbolisation skeleton in these areas) must be extracted, taking into account density constraints. A way of doing this consists in visiting each node of a meshing covering the area and then considering the current node as the starting point of a slope line along which we go down until exiting the steep slope screes area or being nearby another existing line. 
Upper borders in steep slope rocky areas are also required to improve the map readability by illustrating breaks in slopes, considered to be especially dangerous for hikers. These lines are extracted keeping only outline sections of rocky areas with their slope oriented within the area. Besides, they have to be longer than a threshold and their pixels' altitude have to be greater than at least half of their neighbours.

Ridges extraction is also currently tested to improve the map readablity.

\subsection{Method}

In the previous paragraph, we looked over useful data for cartography in mountain areas. Now we need to affect a symbolisation to these data. This is what the user really sees and reads on the map. The whole issue is to represent in the best possible way the terrain complexity. The main point is to understand accurately the map, so that users can not be mistaken and thus avoid dangerous zones for example. When most of users look at the topographic map at a big scale, they trust in its accuracy. This kind of map has to keep this asset.

Besides reflecting the reality, the representation has to be as automatic as possible, as one of the main purposes of this study is to reduce the map production cost. Up to now, rocks have been drawn manually by experienced cartographers, using graphic means and working with aerial photographs. This technique produces very good results but the point is that it is very expensive. (Hurni et al. 2001) has studied some methods to automate the cartographic representation. However, he advocates the combination of manual tasks with automatic ones. According to him, digital methods can be applied only in a limited spectrum of tasks. Control of the full process by an experienced operator is still necessary and desirable, in order to keep the graphic quality that is characteristic of Swiss maps. At IGN France, we wonder as well how we can improve current results in digital cartographic representation. So we have kept carrying out some researches about this issue.

According to several case studies done in mountain areas, hachures seem to be a relevant way for rocks representation. They provide both a good perception relief and an appreciated graphic result. This technique outcomes from a former one more general, used historically to depict the relief with hachures. They were generally drawn along the biggest slope so that the user could mentally think mounts and valleys. In our study case, we judge necessary to have ridge-lines depicting major contours and ridge crests so they complement hachures representation. Maps from the Swiss National Map Series produced by the Federal Office of Topography illus- 
trate this way of representing rocky areas. Fill hachures are plotted manually either along the biggest slope where this one is steep or along contour lines. An operator estimates the slope value looking at aerial photographs. As the human eye is a subjective tool, there is no mathematic threshold to determine if a slope is steep or not.

This way of representing rocky areas has been adapted in this study, trying to incorporate it in a digital process. A hatched pattern has been designed to fill in rocky areas where the slope exceeds the given threshold of $100 \%$. In order to have a fine visual perception, (Imhof 1982) gives in particular some advices dealing with hachures design and related parameters. They have been taken into account to draw up this pattern. Following the different orientation slope classes, this one is always oriented according to the steep slope. For instance, rocky areas with a slope oriented from 0 to $20^{\circ}$ will have a $10^{\circ}$ oriented hatched pattern. Hence the interest to have a classification depending on the slopes orientation. This is necessary to let the pattern follow the neighbour steep slope, whatever the slope orientation value is.

Another hatched pattern has been defined for rocky areas with gentle slopes. In this case, hachures are more spaced. Added points symbols and areas randomly disposed are part of the pattern to mean the presence of isolated rocky blocks. The pattern is oriented following the average tangent to the contour line for each class. The hachures logic spacing used in this case is the same for contour lines. The denser they are, the steeper the slope is, and vice versa.

Hachures are voluntarily irregular to cleave better to the real world. In fact, structure lines and the general rocks texture are hardly ever regular and geometric. Thus, when we had to design a pattern, we paid special attention to the possibility of reproducing it without any spatial discontinuity. An off-setting on the edge of the pattern does not produce a satisfying visual effect. This is especially true if there are no structure lines to hide it.

The screes representation is characterised by points symbols. There are small round points and irregular round shapes that mentally suggest to the map reader stones or rocks. Where there are steep slopes, screes tend to form streams because of unsteady rocks rolling down. These rocks crumbling leave a trace on the ground that can be observed on aerial photographs. We tried to duplicate as precisely as possible those characteristics. Depending on the slope value, we differentiated the screes representation. (Jenny 2008) presents a digital method for scree representation developed at Zürich Institute of Cartography. It gives almost similar results, though there is a difference in his process with shadow areas

Lines along the steepest slope are only extracted in screes areas where the slope rises above 50\%. They are necessary for the representation sup- 
port in these zones. In fact, round points symbols are computed and placed at regular intervals along these lines. The symbols diameter increases in a linear way when we go down along the slope. Visually, the crumbling effect appears thanks to the lines support. They are not visible on the map but we can easily imagine them.

Dealing with screes representation located in slopes under 50\%, a pattern has been drawn. It is made up of punctual symbols and irregular round shapes, and suggests rocks randomly disposed. On the contrary of pattern used in rocky areas, this last is not oriented. This is to keep the randomness of these zones structures.

Upper borders in rocky areas complement the cartography and give a global structure of these mountain areas. They are useful to mean cliff tops and broken grounds. They are drawn with two lines. The first one is black to underline the break; the other one is dark grey to mean the shadow, which is a frequent characteristic within these zones. The black line has a varying width. This is to avoid a symbolisation that would appear too much geometric. Besides structuring the map in mountainous areas, these upper borders warn against potential dangers that may have not been clearly seen with hachures.

Previous listed elements are put together with more classic and often used ones talking about mountain cartography and relief: I mean contour lines and hill shading.
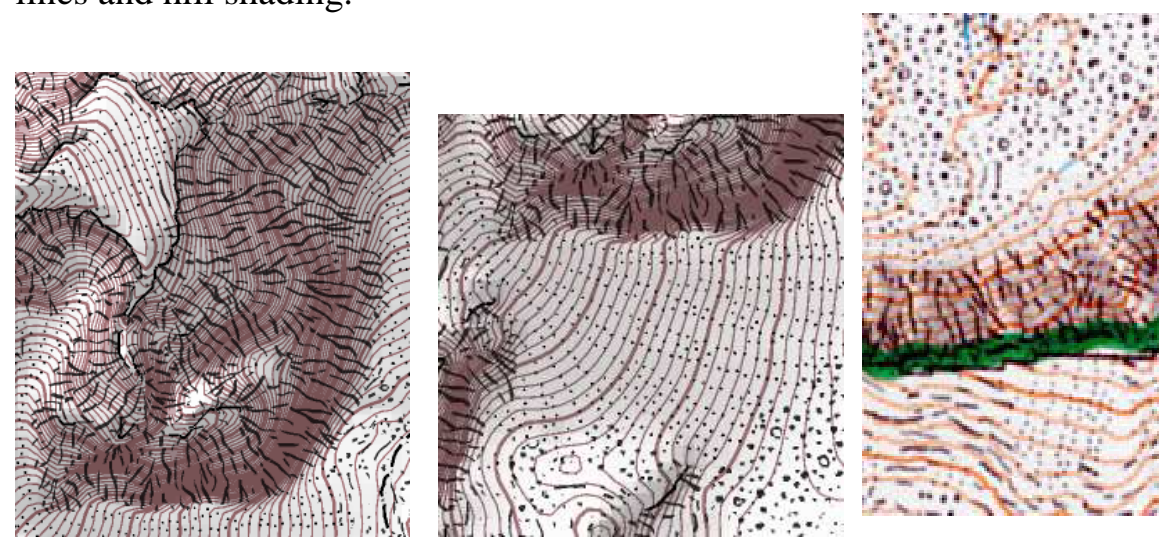

Fig. 5. Representation of rocks and screes depending on slope. 


\subsection{Results}

This representation has been tested in Pyrenees nearby the Ossau peak. More recently, other tests have been done in French Alps, within the Vanoise National Park region nearby Modane.(Figures 7 and 8)

Two softwares have been used to match digital data and obtain a resulting paper map since data were prepared (i.e. imported and georeferenced) thanks to the GIS Geoconcept (produced by Geonconcept SA ltd) before being exported and integrated in Mercator (produced by Star-Apic ltd), a cartographic symbolisation software allowing to manage all data representation with layers in order to do map-printing. This software has been used at IGN France for a long time to manage the end of topographic maps production flowlines, until they are printed.

We have got some results that seem to be hopeful. The map has a good global readability. The whole coherency between themes is satisfying as no layers overrides others. Furthermore, we can easily distinguish the different kind of symbolised zones. The representation rationale and the patterns used here allow the map reader to associate the ground nature. Finally, this is promising all the more that this cartography has been entirely done in a digital way, which was one of the goals.

A visual comparison has been made with the former map manually realised. Besides, some expert users have been asked to compare the two maps. They let us know their feedback. This evaluation enables us in particular to identify a weakness dealing with the digital hill shading. In fact, it is far less expressive than the old one drawn manually. So it still needs to be improved, given that there are a lot of current researches in this field. The cartographic contrasts on the digital map need to be underlined, in order to have a better information structure. For instance, dangerous areas should be perceived directly. Another problem deals with glaciers and the detection of this theme. Depending on when aerial photographs have been taken, snow-covered areas can be still present even in summertime. For the time being, these areas are automatically classified as being glaciers whereas they really correspond to screes or rocky areas. We currently try to find out a solution to this problem.

Of course, this method is to be reproduced on other mountain areas. Remind us that the New Base Map project aims at producing a homogeneous representation on the whole territory, and in particular concerning rocky areas. Currently, this is not the case in France. The result is different and depends on both cartographers and when it has been produced. In order to test the reliability and the scalability of this method, we understand better why this kind of test has to be generalised to other areas. 


\section{Conclusion}

The New Base Map project carried out several studies dealing with image processing and mountain cartography. One of the main purposes is to get an automated digital high mountain cartography as good as the one we had before with manual processes. This study has been done combining several data sources, i.e. orthophotos, DTM and external knowledge, an automated extraction of rocky, screes and glaciers areas, and additional data reliefrelated. These data allow to obtain an automated cartographic representation that aims at improving the relief and the "expression" of high mountain zones. The whole process is described on figure 6 .

The first results obtained in Pyrenees and Alps are hopeful. Further tests could include a customer survey, especially concerning mountain map users. Remarks from customers could help us to improve the final process and particular stages, in particular the hill shading. Then, we might be able to introduce this global solution within the IGN base map production flowline.

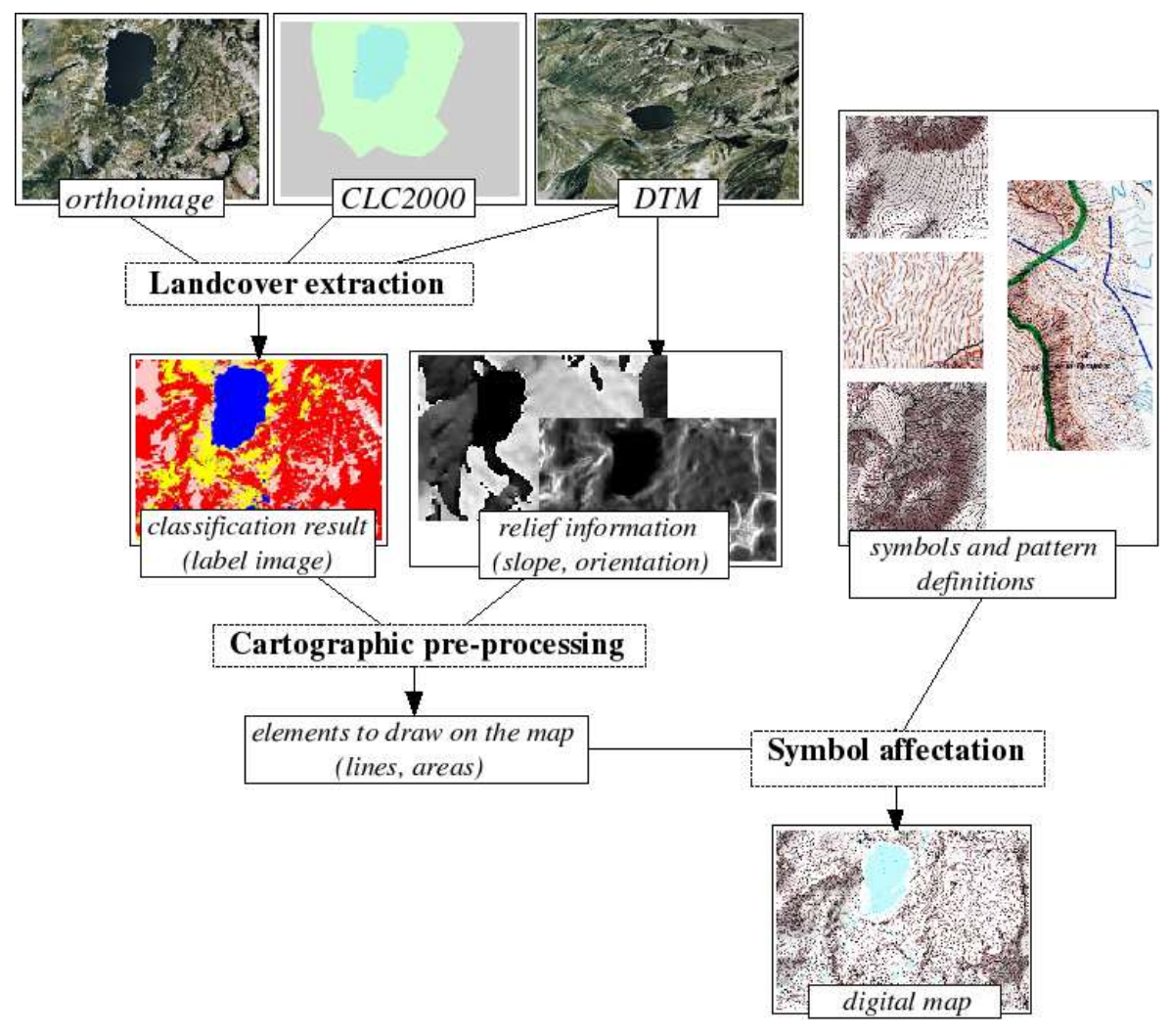


Fig. 6. Description of the whole mountain cartography process.
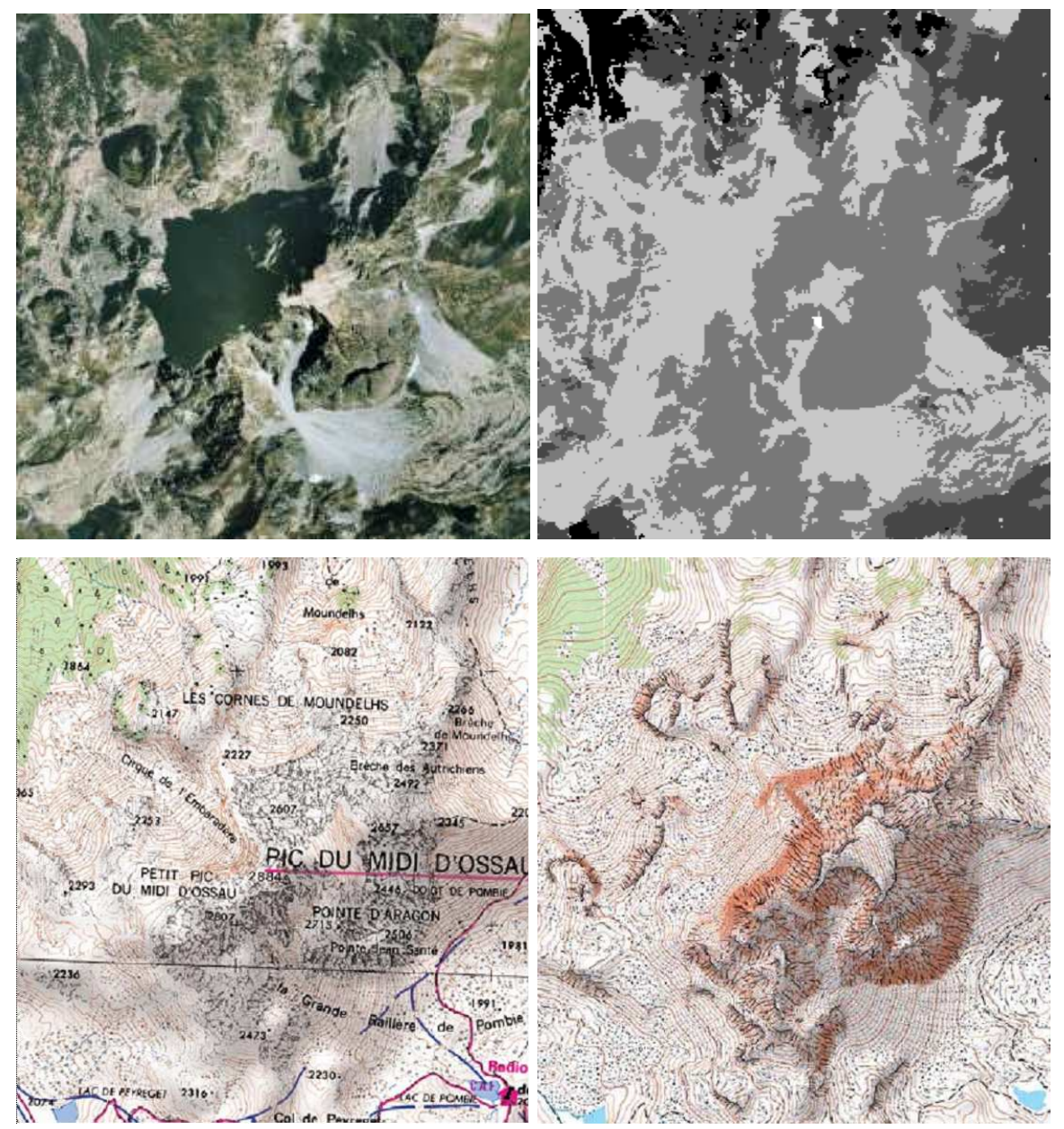

Fig. 7. On the first line, crop of the orthoimage near Ossau-Peak and obtained classification on the same area (from the darkest to the lightest: forest, pasture, rocks, screes and glaciers). On the second line, present handmade topographic map and new fully digital made map on the same area 

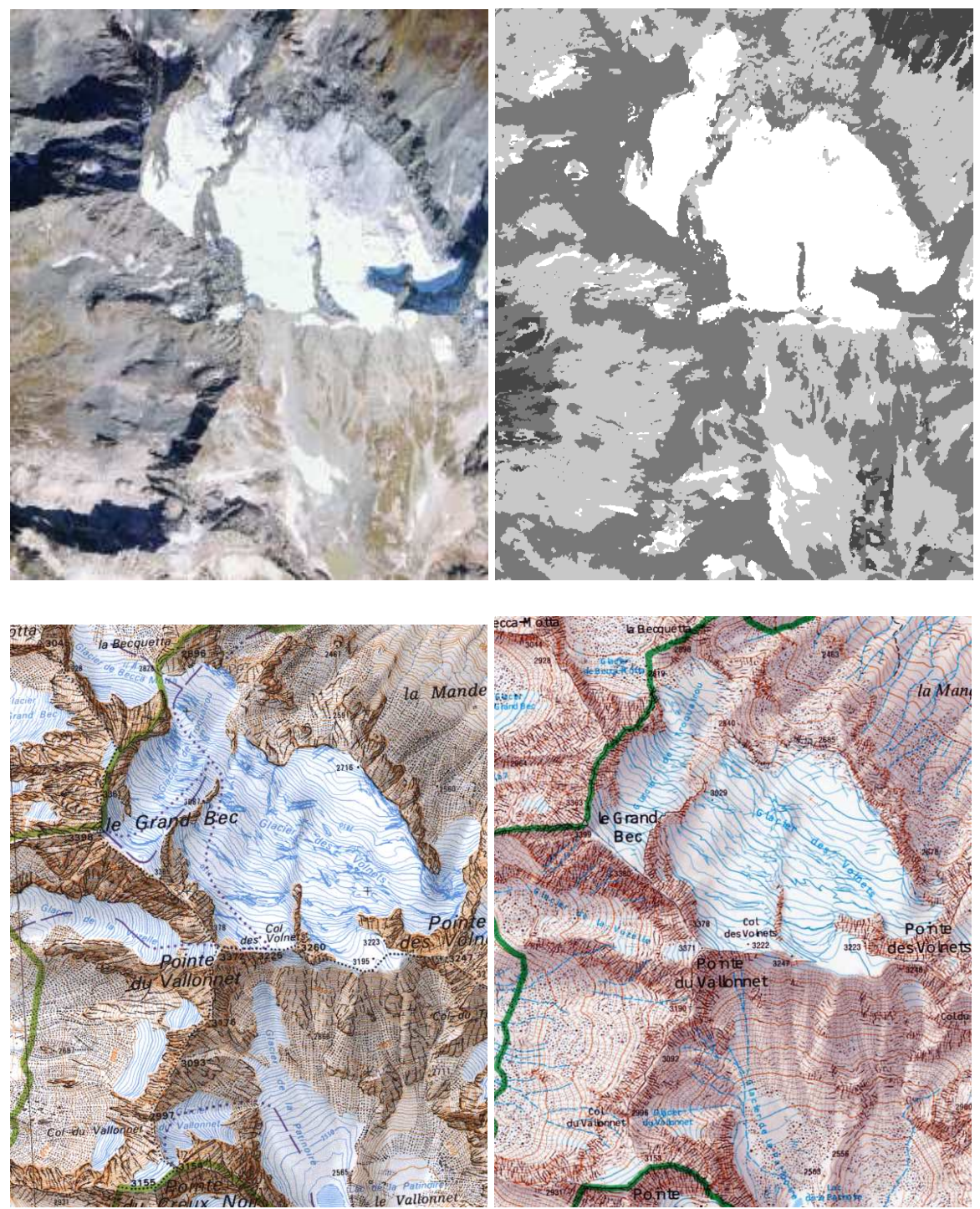

Fig. 8. From left to right: on the first line, crop of the orthoimage near Modane and obtained classification on the same area (from the darkest to the lightest: pasture, rocks, screes and glaciers). On the second line, present handmade topographic map and new fully digital made map on the same area. 


\section{References}

Bossard, M., Feranec, J. and Otahel, J. (2000) CORINE Land Cover technical guide - addendum 2000. technical report $\mathrm{n}^{\circ}$ 40. Technical report, European Environment Agency.

Elhai H. (1968). Biogéographie, Paris: Armand Colin.

Guigues L. (2004). Modèles multi-échelle pour la segmentation d'images, CergyPontoise: Ecole doctorale Sciences et Ingénierie de l'Université de CergyPontoise.

Guigues L., Coquerez J.-P. and Le Men H. (2006). “Scale sets image analysis”, International Journal of Computer Vision, $n^{\circ} 68(3)$.

Hurni L., Dahinden T. and Huztler E. (2001). "Digital Topographic Drawing for Topographic Maps: Tradional Representations by Means of new Technologies", International Publications on Cartography, vol.38, Zurich: Institute of Cartography, Swiss Federal Institute of Technology.

Imhof E. (1982). Cartographic Relief Presentation, W. De Gruyter .

Jenny B. (new release, 2008). Automatic scree representation for topographic maps, Zürich: Institute of Cartography, Swiss Federal Institute of Technology.

Lacambre A. (2001). Aléas et risques naturels en milieu montagnard; apport et limite d'un système d'information géographique, Paris: Université Paris 4.

Le Bris A. and Boldo D. (2007). "Extraction of landcover themes out of aerial orthoimages in mountainous areas using external information", Proc. of the ISPRS Conference Photogrammetric Image Analysis (PIA), 19-21 September, 2007, Munich, 123-128.

Le Men H., Trevisan J., and Boldo D. (2002). "Automatic extraction of landcover themes on digital orthophotos in mountainous area for mapping at $1 / 25 \mathrm{k}$ ", Proc. of the ISPRS Commission II, 20-23 August, 2002, Xi'an, China, 331337.

Paul, F. (2003). The new Swiss Glaciers Inventory: application of remote sensing and GIS. Zürich: Zürich University.

Schwarz, G. (1978). Estimating the dimension of a model. The Annals of Statistics $n^{\circ} 6,461-464$

Trias-Sanz R. (2006). Semi-automatic high-resolution rural landcover classification, Paris: Université Paris 5.

Trias-Sanz R. and Boldo D. (2005). "A high-reliability, high resolution rural land cover classification into forest and non forest", Proc. of the Scandinavian Conference on Image Analysis (SCIA), Lecture notes on computer science, vol. 3540, Finland: Springer, Joensuu, 831-840.

Wang, Z., Ziou, D. and Armenakis, C. (2003). "Combination of imagery - a study on various methods." Proc. of the IEEE International Geoscience and Remote Sensing Symposium (IGARSS), Toulouse, France. 